\title{
Intraspecific Variation and Taxonomic Delimitation of Munronia pinnata (Meliaceae) in Sri Lanka
}

\author{
Dharmadasa R.M. ${ }^{1, *}$, Hettiarachchi P.L. ${ }^{2}$, Premakumara G.A.S. ${ }^{1}$ \\ ${ }^{1}$ Herbal Technology Section, Industrial Technology Institute, Bauddhaloka Mawatha, Colombo 7, Sri Lanka \\ ${ }^{2}$ Department of Biological Sciences, Faculty of Applied Sciences, Rajarata University of Sri Lanka, Mihintale,Sri Lanka \\ *Corresponding author: rathne66@yahoo.com
}

Received February 16, 2014; Revised February 25, 2014; Accepted March 02, 2014

\begin{abstract}
Munronia pinnata (Wall) The ob (Meliaceae) is a rare, therapeutically important medicinal plant used in traditional and folklore medicine in Sri Lanka. Available species/morphotypes/ecotypes of M. pinnata in different locations exhibit diverse range of variations suggesting the possible revision of current taxonomic status. Present study compares seventy different vegetative and reproductive morphological characters in order to suggest the possible taxonomic delimitation of 13 populations of M. pinnata available in Sri Lanka. A cluster analysis and a Principle Component analysis (PCA) were performed using average taxonomic distance. A single access (sequential), key to sub specific taxa was constructed using stable morphological characters. In the cluster, APRG - 5 populations (Population collected from Ritigala forest) has got separated from all the other populations at a distance of about 100\%. Populations collected from the same geographical areas (Gampaha district GPPW - 3, GPWP - 3; Moneragala district MGMG - 9 MGWW - 7; Nuwara Eliya district NEKP - 3, NEMR - 3) have grouped closely either in the dendrogramme or in PCA or both. Based on the results, it could be concluded that population APRG - 5 is distantly related to all others and deserved placing as a sub specific taxon of $M$. pinnata. Further, populations collected from the same geographical area seem to be closely related with one another. Sequential key prepared using morphological characters could be used for the identification of $M$. pinnata populations even in the absence of flowers.
\end{abstract}

\section{Keywords: cluster analysis, intraspecific variation, meliaceae, morphology, munronia pinnata}

Cite This Article: Dharmadasa R.M., Hettiarachchi P.L., and Premakumara G.A.S., "Intraspecific Variation and Taxonomic Delimitation of Munronia pinnata (Meliaceae) in Sri Lanka." World Journal of Agricultural Research, vol. 2, no. 1 (2014): 26-36. doi: 10.12691/wjar-2-1-5.

\section{Introduction}

The Genus Munronia Wight. (Meliaceae), comprising 13 - 15 species, is naturally distributed in China, Sri Lanka, India, Indonesia, Malaysia Vietnam and the Philippines [1,2]. Munronia pinnata (Wall) The ob. is a rare medicinal plant species that occurs in Sri Lanka. Populations with an array of variable phenotypic characters (e.g. compound leaf with leaflet number varying from 3 to 11, different types of leaf margins etc.) are available in various locations in Sri Lanka [3,4,5]. Moreover, earlier literature showed that $M$. pinnata was abundant and widely distributed in Sri Lanka in early days $[1,2]$. Further, all populations growing in Sri Lanka are treated as a single species due to inadequacy of available data for the recognition of infraspecific taxa [2]. It also suggests a correlation between geographical distribution and phenotypic variation in M. pinnata of Sri Lanka. Even though studies on the geographical distribution, comparative pharmacognostic studies of M. pinnata and Andrographis paniculata'[6], preliminary taxonomic status, and the genotypic variation of some populations using Randomly Amplified Polymorphic DNA) are available $[7,8]$, no detailed taxonomic study of $M$. pinnata populations has been carried out. Present study focused on a comprehensive morphological investigation of Sri Lankan populations of $M$. pinnata for elucidating the taxonomic status and developing an identification key.

\section{Materials and Methods}

\subsection{Plant materials}

After getting permission from the relevant authorities, same aged, seed propagated $M$. pinnata plants, were collected from different climatic zones (wet, intermediate and dry zones) of the country (Map 1; Plate $1 \&$ Table 1). These were identified by comparing them with authentic herbarium specimens deposited at National Herbarium. Voucher specimens (HTS1 - HTS13) for all populations were prepared and deposited in the institutional herbarium. As a measure to minimize the impact of environmental factors on the data collected, all plants were potted in the same mixture of potting medium (sand: topsoil: compost $=1: 1: 2$ ) and maintained in the green house under same controlled conditions for five years before taking samples. At least twenty individuals per population were used to record morphological data. 


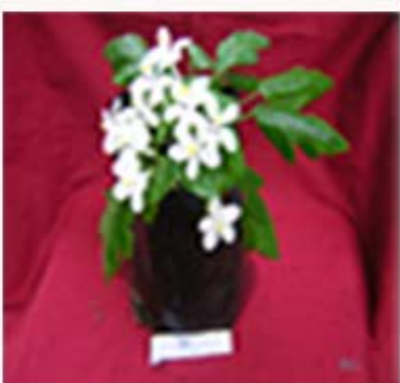

APRG-5

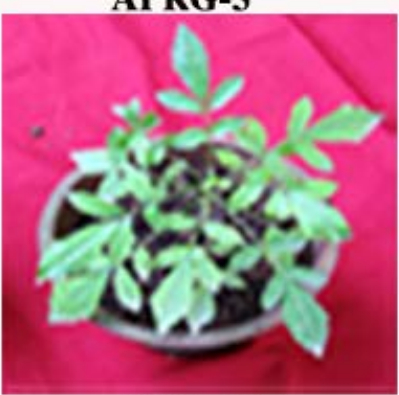

KGKP-5

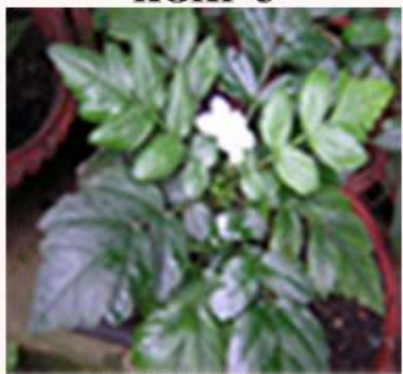

MGWW-7

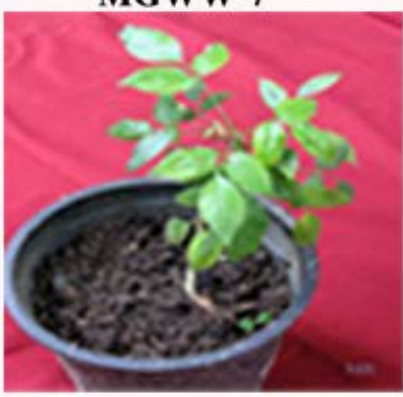

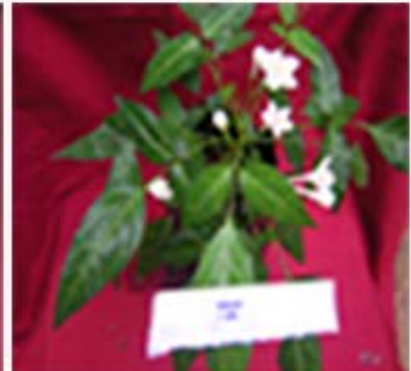

BDHM-3

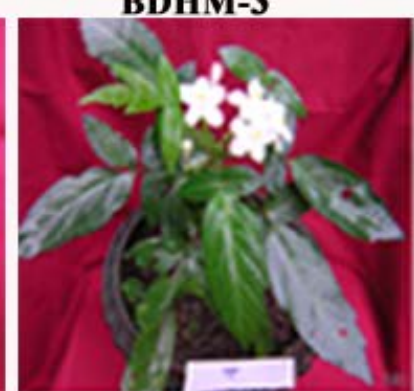

MGMD-3

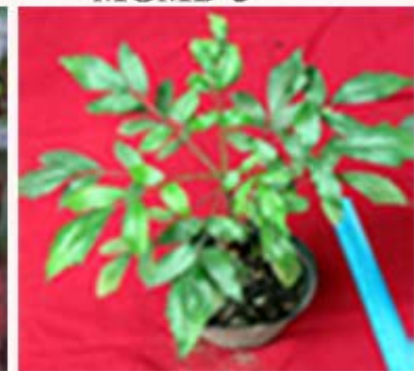

MTMM-5

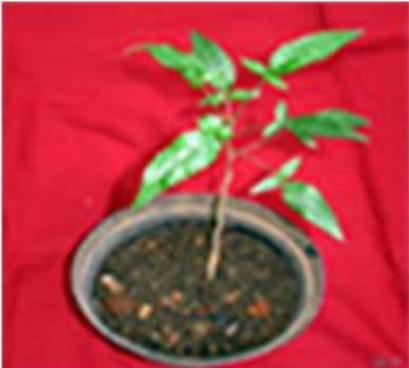

GPPW-3

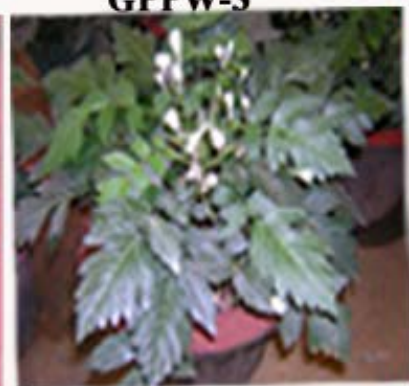

MGMG-9

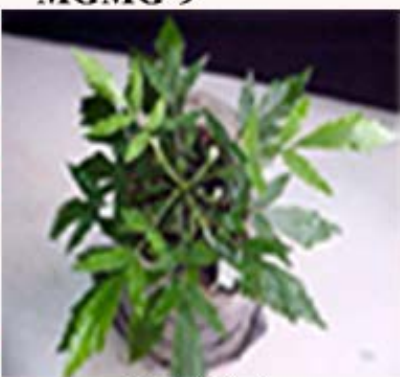

MTNU-5

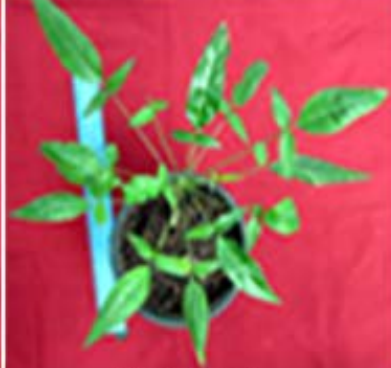

GPWP-3

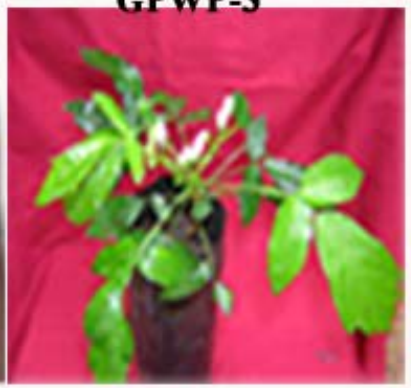

MGNG-3

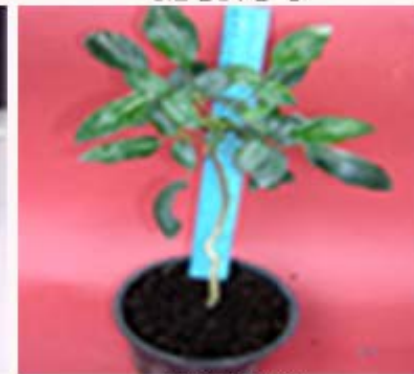

NEKP-3

NEMR-3

Plate 1. Different populations of Munronia pinnata used for the study. [Details of population codes are as given in Table 1. The last number of each code denotes the number of leaflets in the compound leaf of each population]

Table 1. The detailed locations of thirteen populations of Munronia pinnata used for the morphological study

\begin{tabular}{|c|c|c|c|c|c|c|}
\hline Population code & Locality & District & Number of Leaflets & Altitude(M) & Agro- climatic zone & Rainfall(cm) \\
\hline APRG-5 & Ritigala & Anuradhapura & 5 & 396 & DL-3 & $100-150$ \\
\hline BDHM-3 & Haldummulla & Badulla & 3 & 960 & IU-1 & $200-250$ \\
\hline GPPW-3 & Pallewela & Gampaha & 3 & 35 & WL-1 & $350-400$ \\
\hline GPWP-3 & Warakapola & Gampaha & 3 & 125 & WL-1 & $350-400$ \\
\hline KGKP-5 & Kuliyapitiya & Kurunegala & 5 & 30 & DL-1 & $150-200$ \\
\hline MGMD-3 & Madulla & Monaragala & 3 & 196 & IL-1 & $250-300$ \\
\hline MGMG-9 & Srivijayapura & Monaragala & 9 & 220 & IL-1 & $250-300$ \\
\hline MGNG-3 & Nilgala & Monaragala & 3 & 283 & IL2 & $250-300$ \\
\hline MGWW-7 & Wellawaya & Monaragala & 7 & 192 & IL-1 & $150-200$ \\
\hline MTMM-5 & Meemure & Matale & 5 & 420 & WM-2 & $200-250$ \\
\hline MTNU-5 & Naula & Matale & 5 & 375 & WM-2 & $200-250$ \\
\hline NEKP-3 & Kithulpe & Nuwaraeliya & 3 & 720 & WU & $300-350$ \\
\hline NEMR--3 & Mathurata & Nuwaraeliya & 3 & 1050 & WU & $300-350$ \\
\hline
\end{tabular}

DL- Low country dry zone; IU- Up country intermediate zone; WL- Low country wet zone; IL- Low country intermediate zone; WM-Mid country wet zone; WU-Up country wet zone 


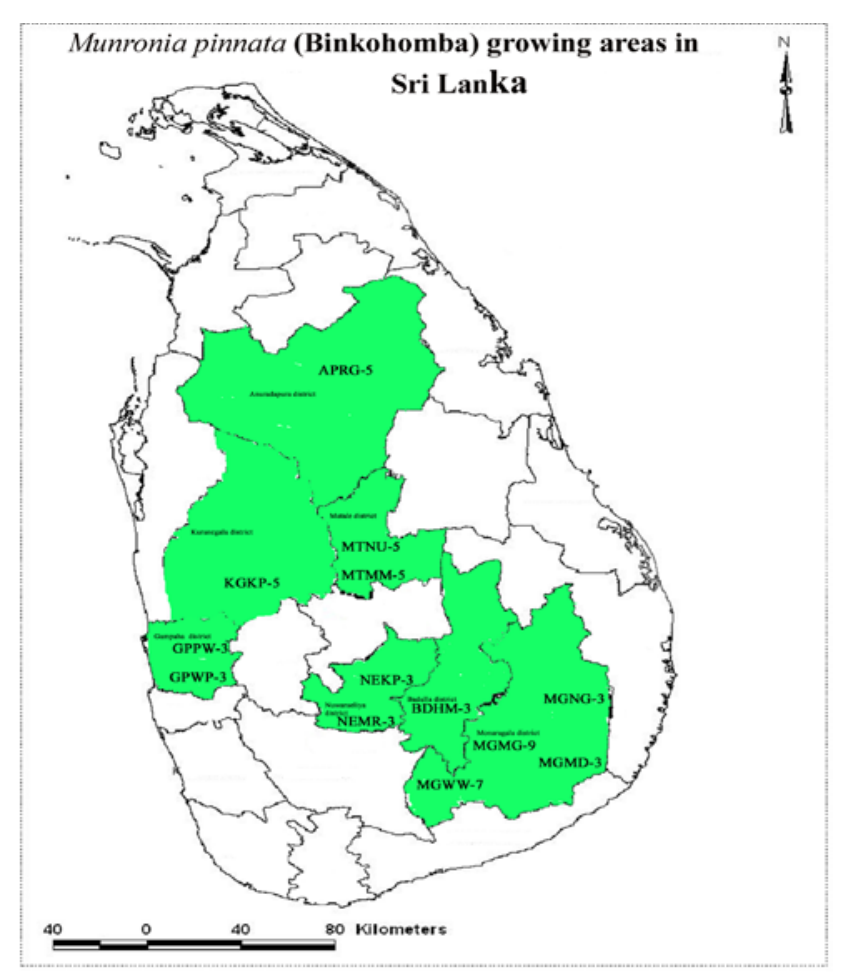

Map 1. Geographical distribution of Munronia pinnata in Sri Lanka [Details of population codes are as given in Table 1. The last number of each code denotes the number of leaflets in the compound leaf of each population]

\subsection{Selecting and Recording Morphological Characters}

Seventy different vegetative and reproductive features, including the characters, which have not been previously recorded, were assessed by using either fresh material or material preserved in $70 \%$ ethanol. The protocols for clearing, staining and mounting of the materials were adopted from literature with slight modifications whenever necessary $[9,10,11]$. Characters were then coded into discrete states as either binary state variables or those with extended variation as multistate variables (Annexure 1 \& Annexure 2) as described by previous studies [12,13]. Photographs of study material were taken using digital microscope (Olympus, Model CX 31, Japan).

\subsection{Cluster Analysis}

The variation range of each character of the plant was divided into two or more character states and ranked them by giving a numerical value for each character state. When interpopulational variation was absent for a given character, data of all individuals of that population were considered as a single value for the data matrix. UPGMA cluster analysis and ordination (correspondence analysis) of the basic data matrix was performed with NTSYS 1.8 [14].

\subsection{Construction of Taxonomic Keys to Identify Munronia pinnata Populations}

A single access (sequential), indented (Yoked) key was constructed using stable morphological characters recorded to identify different populations of $M$. pinnata collected from different localities.

\section{Results and Discussion}

\subsection{Morphological Variation}

Present study identified 25 monomorphic characters of which six were vegetative characters and the rest was reproductive characters, as common features to all populations collected from the three major climatic zones in Sri Lanka. This is acceptable as all these are currently classified under a single species [2]. However, with respect to the rest of the characters, a large interpopulational variation (about $66 \%$ of the characters) was observed among the populations studied supporting the idea of the existence of clearly defined taxa below species level.

As all plants used for the study were acclimatized for at least five years under same soil and climatic conditions, the morphological characters recorded could be considered as a true reflection of genetically determined morphology of the plants concerned. An intra specific monomorphism in some common floral characters was evident in this study supporting the existing classification, which considers all morphotypes as a single species. In contrast, a considerable level of polymorphism was also found in some vegetative characters and in fine floral characters such as stamina tube length, stigma shape and style length. Some of these seem very promising in differentiating populations.

All characters used in the key are vegetative and therefore, could be used even in the non-flowering season of the plant. Since flowering is very rare in certain populations [3], it is essential to prepare a key based on vegetative characters. Moreover, these plants were maintained under the same environmental conditions for 5 years and hence, can be considered as very stable and highly reliable taxonomic features of the plant.

The principle component analysis shows that 3leaflet bearing types (except GPPW - 3 \&GPWP - 3) are well separated from rest of the populations at a distance of $80-85 \%$ in the $3^{\text {rd }}$ cluster (Figure 1 \& Figure 2). Interestingly, there is a close genetic relatedness among the populations collected from the same geographical area [(MGMD -3 and MGNG - 3 from intermediate zone (IL); GPPW - 3 \& GPWP - 3 from low country wet zone (WL); NEKP - 3, NEMR - 3 from up country wet zone (WU)]. The presence of phenotypic variation, which could be correlated with the geographical distribution, was suggested by previous studies and results of the present study have proved it [2].

As shown in Figure 1 \& Figure 2, the population collected from Ritigala area (APRG - 5) has got well separated from other populations at a distance of $95 \%$ in the cluster and along the first axis at one extreme end (left side) in principle component analysis. This is acceptable as it bears several unique characters such as undulate distal leaf margin, crenate proximal leaf margin, wide leaves, droopy plants, exceptionally high vein islet number, veinlet termination number, low number of flowers per inflorescence, a globose shape 
style base and a very low number of seeds per fruit. The microclimate surrounding Ritigala reserve forest is very much different from that in other locations. This might have favored the growth and survival of APRG - 5 population. These Geographically isolated populations tend to become genetically specific due to the absence of mixing of genes with other populations. The phenetic distance observed between APRG - 5 population and other populations in the dendrogramme support the idea that this population has diverged in another direction. In the process of evolution, because of isolation and adaptation, vegetative parts of organism change prior to reproductive parts. Therefore, observations of this study are in accordance with the natural process of speciation and evolution and strengthen the idea of placing APRG - 5 population in a separate sub specific taxon of $M$. pinnata. A broader comparison with respect to characters of other disciplines such as micromolecules, proteins and DNA might help to decide the exact position or taxonomic status (whether it has to be a different variety or sub species). Presence of phenotypic variation, which could be correlated with the geographical distribution, was suggested in the Flora of Ceylon and results of this research have proved it. Further, the observed separation of the 3 - leaflet group was similar to the findings reported by previous workers who analyzed eight morphological characters together with some DNA evidence in five populations of $M$. pinnata [7].

Populations collected from Gampaha district (GPPW - 3, GPWP - 3) and Moneragala district (MGMD - 3, MGNG - 3) have grouped closely in dendrogramme. Clustering of populations GPPW - 3, GPWP - 3 is acceptable as these populations bear many similar morphological features such as the habit of the plant (medium sized plants), smooth shiny texture of the leaves, terminal leaflet length, shape of the terminal leaflet base (acute), leaf margin (entire), and proximal leaflet margin (undulate). It shows that, populations collected from the same area have some kind of genetic relationship, which might not be noticeable at once when we consider the morphological traits in these populations.

Populations bearing more than three leaflets except NEKP-3 got separated from others at a distance of about $75 \%$ (Cluster $3 b$ ). In the cluster, NEKP - 3 has got separated from other populations at a distance of about 80\%. Since all 3- leaflet types were clustered together, in both dendrogramme and Principle component analysis, it clearly indicates a close genetic relatedness among 3 - leaflet bearing populations (Figure 1 \& Figure 2).

\subsection{Sequential Indented Key Based on Vegetative Morphology}

Leads of each couplet of sequential indented key prepared give reliable characters, which could be used to differentiate very closely resembling populations of M. pinnata.

All characters used in the key are vegetative and therefore, could be used even in the non-flowering season of the plant. Since flowering is very rare in certain populations [3], it is essential to prepare a key based on vegetative characters. Moreover, these plants were maintained under the same environmental conditions for 5 years and hence, can be considered as very stable and highly reliable taxonomic features of the plant.

The principle component analysis shows that 3 leaflet bearing types (except GPPW - 3 \&GPWP - 3) are well separated from rest of the populations at a distance of $80-85 \%$ in the third cluster (Figure 1 \& Figure 2). Interestingly, there is a close genetic relatedness among the populations collected from the same geographical area [(MGMD - 3 and MGNG - 3 from intermediate zone (IL); GPPW - 3 \& GPWP - 3 from low country wet zone (WL); NEKP - 3, NEMR - 3 from up country wet zone (WU)]. The presence of phenotypic variation, which could be correlated with the geographical distribution, was suggested by previous studies and results of the present study have proved it [2].

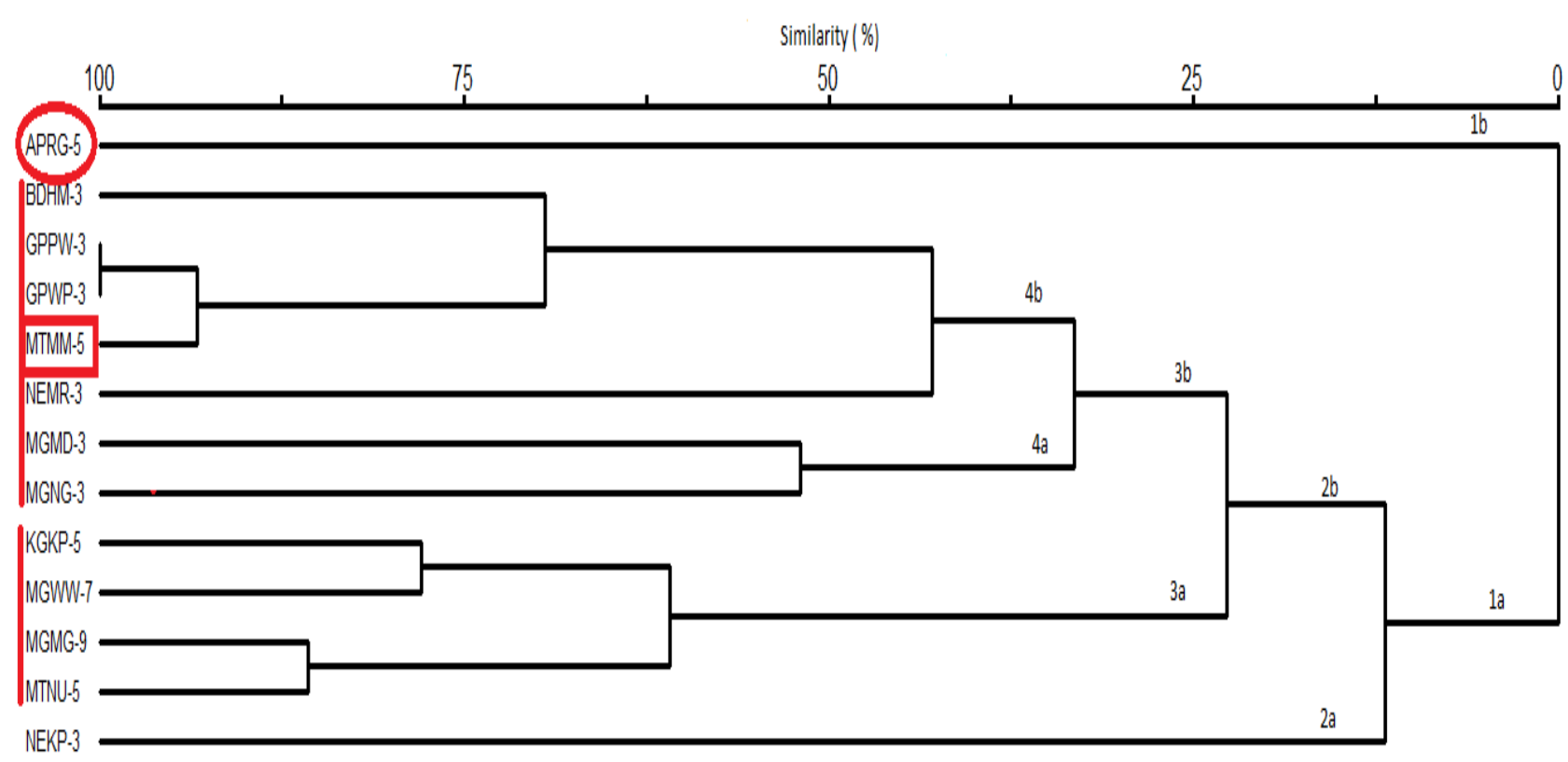

Figure 1. Dendrogramme generated by combining 45 polymorphic vegetative and reproductive characters of 13 populations of Munronia pinnata 


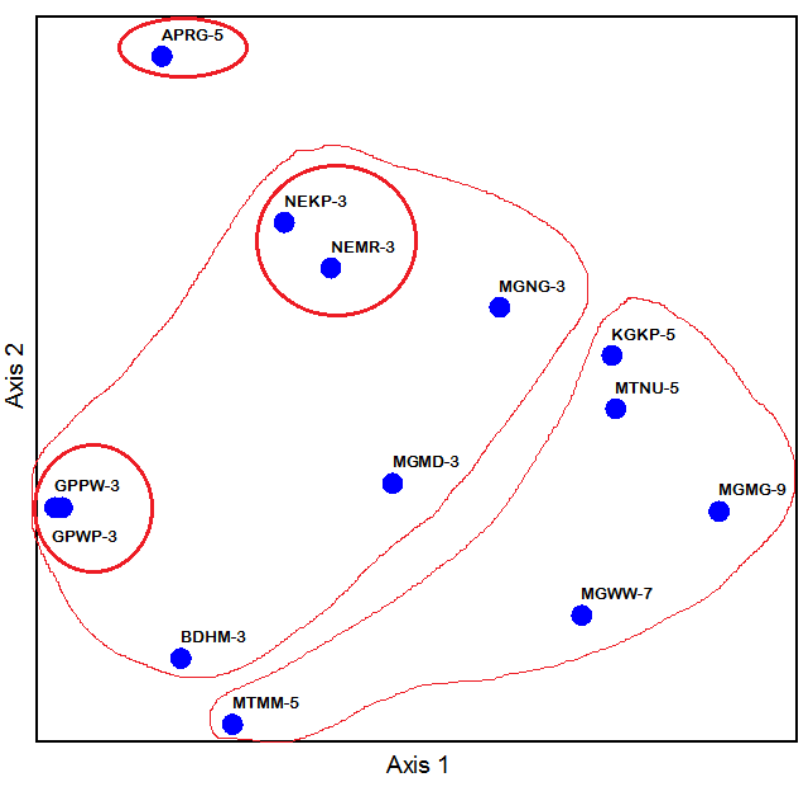

Figure 2. Principle Component analysis on basis of 45 polymorphic vegetative and reproductive characters of 13 populations of Munronia pinnata

\subsection{Sequential Indented Key Prepared to Identify Different Populations of Munronia pinnata}

1. Number of leaflets three ...GPWP-3, NEMR-3, NEKP-3, MGNG-3, BDHM-3, MGMD-3, GPPW-3

2. End leaf margin entire...EMR-3, NEKP-3, BDHM-3, GPWP-3

3. Side leaflet base oblique ...NEMR-3

3. Side leaflet base not oblique...NEKP-3,

GPWP-3, BDHM-3

4. End leaflet veins $14 \ldots$ BDHM-3

4. End leaflet veins less than $14 \ldots$ NEKP-3, GPWP-3

5. Side leaflet margin undulate.... GPWP-3

5. Side leaflet margin not undulate ...

2. End leaflet margin not entire...MGMD-3, MGNG-3, GPPW-3

6. End leaflet veins less than 10 ...MGNG-3

6. End leaflet veins more than 10 MGMD-3, GPPW-3

7. Side leaflet primary-secondary vein angle at base $45-60^{\circ} \ldots \ldots \ldots$.......PW- 3

7. Side leaflet primary-secondary vein angle less than $45-60^{\circ} \ldots . . \mathrm{MGMD}-3$

1. Number of leaflets more than three......APRG-5, MTMM-5, MTNU-5, MGMG-9, MGWW-7, KGKP-5 8. Secondary - tertiary vein angle less than $60^{\circ} \ldots$ MTMM-5

8 . Secondary-tertiary vein angle not less

than $60^{\circ} \ldots$ APRG-5, MTNU-5, MGMG-9. MGWW-7, KGKP-5

9. End leaflet veins less than 10 ...MTNU-5

9. End leaflet veins more than 10 APRG-5,

MGMG-9, MGWW-7, KGKP-5

10. End leaf margin not dentate....

APRG-5

10. End leaf margin dentate ...MGMG-9,

MGWW-7, KGKP-5

11. Side leaflet apex acute- MGMG-9

11. Side leaflet apex not acute..

MGWW-7, KGKP-5

12. Side leaflet base

rounded .....MGWW-7

12. Side leaflet base not

rounded ............. KGKP-5

\section{Conclusions}

Population collected from Ritigala forest (APRG-5) is distantly related to all others and deserved placing as a sub specific taxon of $M$. pinnata. Further, populations collected from the same geographical area (Gampaha and Moneragala districts) seem to be closely related with one another $M$. pinnata populations could be identified even in the absence of flowers using the key constructed in the study.

\section{Acknowledgement}

Financial support given by institutional grants is greatly appreciated.

\section{References}

[1] Hooker, J.D. 1872. Flora British India Vol. 2.Published under authority of the secretary for India for state council. Savills Edwards and co, Printers, Chandos street, covert garden London. 542-543.

[2] Mabberley, 1995. Meliaceae. In: Dassanayake MD, Forsberg F.R. and Clayton WD. eds. A Revised Handbook to the Flora of Ceylon. Vol ix. Amerind Pub. Co. Ltd. New Delhi 230-239.

[3] Dharmadasa, R.M., Hettiarachchi, P.L. and Premakumara, G.A.S. 2011. Geographical distribution and conservation of a rare medicinal Plant Munronia pinnata (Wall.) Theob.(Meliaceae) in Sri Lanka. Bangladesh J. Plant. Taxon. 18 (1): 39-49.

[4] Dassanayake, M.D, Balasubramanium, S. 1995. A preliminary study of secondary metabolites in selected families. Proc. 51st Annual Session, Sri Lanka Association for the Advancement of Science, Sri Lanka, 127.

[5] Jayaweera B.M.A. 1981. Medicinal plants (indigenous and exotic) used in Ceylon- part (iv). Natn. Sci. Coun. Sri Lanka. 59.

[6] Dharmadasa, R.M. Samarasinghe K, Adhihetty P, and Hettiarachchi P.L. (2013) Comparative Pharmacognostic Evaluation of Munronia Pinnata (Wall.) Theob. (Meliaceae) and Its Substitute Andrographis paniculata (Burm.f.) Wall. Ex Nees (Acanthaceae). World Journal of Agricultural Research 1, No. 5: 77-81.

[7] Ruckshanthi, J.P.D, Pushpakumara, D.K.N.G, Perera, A.L.T, Muhunthan, R. 2002. Morphological and genetic (RAPDs) characterization of M. pinnata (Meliaceae). Tropical Agricultural Research 14: 11-21.

[8] Hettiarachchi, P.L, Dharmadasa, R.M, Premakumara, G.A.S, Perera, A.M.M.S. and Hettiarachchi, S. 2007. A Preliminary study of taxonomic status of Munronia pinnata (Wall) Theob. (Meliaceae) in proceeding, 27th Annual Session of Iinstitute of Biology, Sri Lanka. 36.

[9] Johansen D.A. 1940. Plant micro techniques McGraw-Hill book company, New York, pp 49-95.

[10] Radford A.E, Dickson W. C, Massey J.R and Bell C.R. 1974. Vascular Plant Systematic. Harper and Row Publishers. New York 891.

[11] Herath T. 1979. Teaching materials for angiosperm taxonomy and micro techniques. Department of botany, University of Colombo, Sri Lanka.

[12] Dilcher D.L. 1974. Approaches to the identification of angiosperm leaf remains. The botanical Review. Department of Plant Sciences, Indiana University, Bloomington, Indiana 40: 184.

[13] Yakandawala D.M.D. 2001. Teaching material for Plant Taxonomy I - Basic Terminology. Department of Botany, University of Peradeniya.

[14] Rolfh FJ. 1993 NTSYS - pc - Numerical Taxonomy and Multivariate Analysis System Version 1.80. Applied Biostatistics Inc. 


\section{Annexures}

\section{Annexure 1}

Table 2. List of morphological characters together with their character states recorded in Munronia pinnata

\begin{tabular}{|c|c|}
\hline Character & Character state \\
\hline \multicolumn{2}{|c|}{ A. Vegetative characters } \\
\hline Plant habit & $\begin{array}{c}\text { Droopy (1), Erect (2), Intermediate } \\
\text { (3) }\end{array}$ \\
\hline Nature of the plant & Robust (1), Medium (2), Small (3) \\
\hline Plant height (cm) & $\begin{array}{c}<10(1), 10-14 \text { (2), } 15-19 \text { (3), }>20 \\
\text { (4) }\end{array}$ \\
\hline No. of leaflets & $3(1), 5(2), 7$ (3), 9-11 (4) \\
\hline Stem trichomes & Present (1) \\
\hline Stem trichome type & $\begin{array}{c}\text { Glandular (1), simple unicellular } \\
\text { (2), bifurcate (3) }\end{array}$ \\
\hline Phyllotaxy & Whorled (1) \\
\hline Leaf venation & Pinnately reticulate (1) \\
\hline Leaf texture & $\begin{array}{l}\text { Velvet (1), Smooth shiny (2), } \\
\text { Rough (3) }\end{array}$ \\
\hline Leaf type & Imparipinnate (1) \\
\hline Petiole length $(\mathrm{cm})$ & $<4(1), 4-6(2),>6(3)$ \\
\hline Leaf length $(\mathrm{cm})$ & $<10(1), 10-15(2),>15(3)$ \\
\hline Leaf width (cm) & $<5(1), 5-7(2),>7(3)$ \\
\hline Leaf L/W ratio & $1-2(1), 2.1-3(2),>3(3)$ \\
\hline Terminal leaflet length (cm) & $<6(1), 6-8(2),>8(3)$ \\
\hline Terminal leaflet width $(\mathrm{cm})$ & $<3(1),>3(2)$ \\
\hline Terminal leaflet L/W ratio & $1-2(1), 2.1-3(2),>3(3)$ \\
\hline Terminal leaflet apex & Acute (1), Obtuse (2) \\
\hline Terminal leaflet base & Rounded (1), Oblique (2) \\
\hline Terminal leaflet margin & $\begin{array}{l}\text { Entire (1), Dentate end leaf lobed } \\
\text { (2),Undulate (3) }\end{array}$ \\
\hline $\begin{array}{c}\text { Number of veins in terminal } \\
\text { leaflet }\end{array}$ & $<10(1), 10-12(2),>12$ (3) \\
\hline Proximal leaflet length $(\mathrm{cm})$ & $3-4(1), 4.1-5.1(2)$ \\
\hline Proximal leaflet width $(\mathrm{cm})$ & $<2(1), 2(2),>2(3)$ \\
\hline Proximal leaflet L/W ratio & $1-2(1),>2(2)$ \\
\hline Proximal leaflet apex & Acute (1), Obtuse (2) \\
\hline Proximal leaflet base & Rounded (1), Oblique (2) \\
\hline Proximal leaflet margin & $\begin{array}{l}\text { Entire (1), Dentate (2), Undulate } \\
\text { (3) crenate (4) }\end{array}$ \\
\hline Proximal leaflet petiole & Prominent (1), Not prominent (2) \\
\hline $\begin{array}{c}\text { Number of veins in proximal } \\
\text { leaflet }\end{array}$ & $<8$ (1), 8 (2), >8 (3) \\
\hline Stipules & Absent (1) \\
\hline Leaf trichomes & Present (1) \\
\hline $\begin{array}{l}\text { P. vein to secondary vein angle } \\
\text { (base) }\end{array}$ & $<450,(1), 45-600(2)$ \\
\hline $\begin{array}{l}\text { P. vein to secondary vein angle } \\
\text { (apex) }\end{array}$ & $<450,(1), 45-600(2)$ \\
\hline S. vein to tertiary vein angle & $45-600(1), 600-900(2),>900(3)$ \\
\hline Areole type & Polygonal (1) \\
\hline Vein islet No. & $\begin{array}{c}0.5-1(1), 1.1-1.5(2), 1.6-2(3), 2.1- \\
2.5(4), 2.5<(5)\end{array}$ \\
\hline Vein termination No. & $<3.5(1), 3.6-.5(2),>5(3)$ \\
\hline \multicolumn{2}{|c|}{ B. Reproductive characters } \\
\hline $\begin{array}{c}\text { Number of } \\
\text { flowers/inflorescence }\end{array}$ & $<4(1), 4(2),>4(3)$ \\
\hline Corolla type & Salver form (1) \\
\hline Flower color & white (1) \\
\hline Flower symmetry & Actinomorphic (1) \\
\hline Corolla length (cm) & $<2.5(1), 2.5-3(2)$ \\
\hline Pedicel & Present (1) \\
\hline Number of sepals & $5(1)$ \\
\hline Sepal trichome upper surface & Present (1) \\
\hline Sepal trichome lower surface & Present (1) \\
\hline Sepal length (mm) & $<6(1),>6(2)$ \\
\hline Sepal width (mm) & $<1.5(1),>1.5(2)$ \\
\hline Number of petals & $5(1)$ \\
\hline Petal length $(\mathrm{cm})$ & $<1.5,(1) 1.5-2(2),>2(3)$ \\
\hline Sepal L/W ratio & $2-4.9(1), 5-7(2)$ \\
\hline Petal width (cm) & $<0.5,(1) 0.5-0.7(2),>0.7(3)$ \\
\hline Petal L/W ratio & $1.5-2.5$ (1), 2.6-3.5 (2), 3.5< (3) \\
\hline Flower length & $2.5-3(1), 3.1-3.5(2), 3.6<(3)$ \\
\hline Petal apex & Acute (1), Truncate (2), Rounded \\
\hline
\end{tabular}

\begin{tabular}{|c|c|}
\hline & (3) \\
\hline Petal venation & Pinnatly reticulate (1) \\
\hline Petal upper surface trichomes & Absent (1) \\
\hline Petal lower surface trichomes & Dense (1) \\
\hline Petal margin trichomes & Present (1), Absent (2) \\
\hline Androceum type & Monodelphous (1) \\
\hline Staminal tube length $(\mathrm{cm})$ & $2>(1), 2-2.5(2),>2.5$ \\
\hline Anther filament attachment & Dorsifixed (1) \\
\hline Anther collar attachment & Double cordate (1) \\
\hline Style length $(\mathrm{cm})$ & $: 2-2.5(1), 2.6-3(2)$ \\
\hline Anther apex & Mucronate (1) \\
\hline Stigma shape & Globose (1) \\
\hline Style length $(\mathrm{cm})$ & $<2.5(1),>2.5(2)$ \\
\hline Stigma hairs & Absent (1) \\
\hline Ovary & Superior (1), Inferior (2) \\
\hline Number of carpels & $5(1)$ \\
\hline Placentation & Axilliary (1) \\
\hline Fruit type & Capsule (1) \\
\hline Number of seeds per fruit & $<7(1), 7-9(2),>9(3)$ \\
\hline Seed colour & Brown (1) \\
\hline Seed length (mm) & $<4(1),>4(2)$ \\
\hline Seed width (mm) & $<2(1),>2(2)$ \\
\hline Seed L/W ratio & $1-2(1),>2(2)$ \\
\hline
\end{tabular}

Table 3. List of morphological characters together with their character states recorded in Munronia pinnata

\begin{tabular}{|c|c|}
\hline Character & Character state \\
\hline \multicolumn{2}{|c|}{ A. Vegetative characters } \\
\hline Plant habit & $\begin{array}{c}\text { Droopy (1), Erect (2), Intermediate } \\
\text { (3) }\end{array}$ \\
\hline Nature of the plant & Robust (1), Medium (2), Small (3) \\
\hline Plant height (cm) & $\begin{array}{c}<10(1), 10-14(2), 15-19(3),>20 \\
(4)\end{array}$ \\
\hline No. of leaflets & 3 (1), 5 (2), 7 (3), 9-11 (4) \\
\hline Stem trichomes & Present (1) \\
\hline Stem trichome type & $\begin{array}{c}\text { Glandular (1), simple unicellular } \\
\text { (2), bifurcate (3) }\end{array}$ \\
\hline Phyllotaxy & Whorled (1) \\
\hline Leaf venation & Pinnately reticulate (1) \\
\hline Leaf texture & $\begin{array}{l}\text { Velvet (1), Smooth shiny (2), } \\
\text { Rough (3) }\end{array}$ \\
\hline Leaf type & Imparipinnate (1) \\
\hline Petiole length $(\mathrm{cm})$ & $<4(1), 4-6(2),>6(3)$ \\
\hline Leaf length $(\mathrm{cm})$ & $<10(1), 10-15(2),>15(3)$ \\
\hline Leaf width (cm) & $<5(1), 5-7(2),>7(3)$ \\
\hline Leaf L/W ratio & $1-2(1), 2.1-3(2),>3(3)$ \\
\hline Terminal leaflet length $(\mathrm{cm})$ & $<6(1), 6-8(2),>8(3)$ \\
\hline Terminal leaflet width $(\mathrm{cm})$ & $<3(1),>3(2)$ \\
\hline Terminal leaflet L/W ratio & $1-2(1), 2.1-3(2),>3(3)$ \\
\hline Terminal leaflet apex & Acute (1), Obtuse (2) \\
\hline Terminal leaflet base & Rounded (1), Oblique (2) \\
\hline Terminal leaflet margin & $\begin{array}{l}\text { Entire (1), Dentate end leaf lobed } \\
\text { (2),Undulate (3) }\end{array}$ \\
\hline $\begin{array}{c}\text { Number of veins in terminal } \\
\text { leaflet }\end{array}$ & $<10(1), 10-12(2),>12(3)$ \\
\hline Proximal leaflet length $(\mathrm{cm})$ & 3-4 (1), 4.1-5.1 (2) \\
\hline Proximal leaflet width $(\mathrm{cm})$ & $<2(1), 2(2),>2(3)$ \\
\hline Proximal leaflet L/W ratio & $1-2(1),>2(2)$ \\
\hline Proximal leaflet apex & Acute (1), Obtuse (2) \\
\hline Proximal leaflet base & Rounded (1), Oblique (2) \\
\hline Proximal leaflet margin & $\begin{array}{l}\text { Entire (1), Dentate (2), Undulate } \\
\text { (3) crenate (4) }\end{array}$ \\
\hline Proximal leaflet petiole & Prominent (1), Not prominent (2) \\
\hline $\begin{array}{c}\text { Number of veins in proximal } \\
\text { leaflet }\end{array}$ & $<8(1), 8(2),>8(3)$ \\
\hline Stipules & Absent (1) \\
\hline Leaf trichomes & Present (1) \\
\hline $\begin{array}{l}\text { P. vein to secondary vein angle } \\
\text { (base) }\end{array}$ & $<450,(1), 45-600(2)$ \\
\hline $\begin{array}{l}\text { P. vein to secondary vein angle } \\
\text { (apex) }\end{array}$ & $<450,(1), 45-600(2)$ \\
\hline S. vein to tertiary vein angle & $45-600(1), 600$ - $900(2),>900(3)$ \\
\hline Areole type & Polygonal (1) \\
\hline Vein islet No. & $\begin{array}{c}0.5-1(1), 1.1-1.5(2), 1.6-2(3), 2.1- \\
2.5(4), 2.5<(5)\end{array}$ \\
\hline Vein termination No. & $<3.5(1), 3.6-.5(2),>5(3)$ \\
\hline
\end{tabular}




\begin{tabular}{|c|c|}
\hline \multicolumn{2}{|c|}{ B. Reproductive characters } \\
\hline $\begin{array}{c}\text { Number of } \\
\text { flowers/inflorescence }\end{array}$ & $<4$ (1), 4 (2), $>4$ (3) \\
\hline Corolla type & Salver form (1) \\
\hline Flower color & white (1) \\
\hline Flower symmetry & Actinomorphic (1) \\
\hline Corolla length (cm) & $<2.5(1), 2.5-3(2)$ \\
\hline Pedicel & Present (1) \\
\hline Number of sepals & Present (1) \\
\hline Sepal trichome upper surface & Present (1) \\
\hline Sepal trichome lower surface & $<6(1),>6(2)$ \\
\hline Sepal length (mm) & $<1.5(1),>1.5(2)$ \\
\hline Sepal width (mm) & $5(1)$ \\
\hline Number of petals & $<1.5,(1) 1.5-2(2),>2(3)$ \\
\hline Petal length (cm) & $2-4.9(1), 5-7(2)$ \\
\hline Sepal L/W ratio & $<0.5,(1) 0.5-0.7(2),>0.7(3)$ \\
\hline Petal width (cm) & $1.5-2.5(1), 2.6-3.5(2), 3.5<(3)$ \\
\hline Petal L/W ratio & $2.5-3(1), 3.1-3.5(2), 3.6<(3)$ \\
\hline Flower length & Acute (1), Truncate (2), Rounded \\
\hline Petal apex & Pinnatly reticulate (1) \\
\hline Petal venation & Table 4. Morphologil character tab \\
\hline &
\end{tabular}

\begin{tabular}{|c|c|}
\hline Petal upper surface trichomes & Absent (1) \\
\hline Petal lower surface trichomes & Dense (1) \\
\hline Petal margin trichomes & Present (1), Absent (2) \\
\hline Androceum type & Monodelphous (1) \\
\hline Staminal tube length (cm) & $2>(1), 2-2.5(2),>2.5$ \\
\hline Anther filament attachment & Dorsifixed (1) \\
\hline Anther collar attachment & Double cordate (1) \\
\hline Style length (cm) & $: 2$-2.5 (1), 2.6-3(2) \\
\hline Anther apex & Mucronate (1) \\
\hline Stigma shape & Globose (1) \\
\hline Style length (cm) & $<2.5(1),>2.5(2)$ \\
\hline Stigma hairs & Absent (1) \\
\hline Ovary & Superior (1), Inferior (2) \\
\hline Number of carpels & $5(1)$ \\
\hline Placentation & Axilliary (1) \\
\hline Fruit type & Capsule (1) \\
\hline Number of seeds per fruit & $<7(1), 7-9(2),>9(3)$ \\
\hline Seed colour & Brown (1) \\
\hline Seed length (mm) & $<4(1),>4(2)$ \\
\hline Seed width (mm) & $<2(1),>2(2)$ \\
\hline Seed L/W ratio & $1-2(1),>2(2)$ \\
\hline \multicolumn{2}{|c}{}
\end{tabular}

\begin{tabular}{|c|c|c|c|c|c|c|c|c|c|c|c|c|c|}
\hline \multirow{2}{*}{$\begin{array}{l}\text { Character } \\
\text { Vegetativ } \\
\text { e } \\
\text { characters }\end{array}$} & \multicolumn{13}{|c|}{ POPULATION } \\
\hline & $\begin{array}{l}\text { APRG- } \\
5\end{array}$ & $\begin{array}{c}\text { BDHM } \\
-3\end{array}$ & $\begin{array}{c}\text { GPPW } \\
-3\end{array}$ & $\begin{array}{c}\text { GPWP } \\
-3\end{array}$ & $\begin{array}{c}\text { KGKP } \\
-5\end{array}$ & $\begin{array}{l}\text { MGM } \\
\text { D-3 }\end{array}$ & $\begin{array}{l}\text { MGM } \\
\text { G-9 }\end{array}$ & $\begin{array}{l}\text { MGN } \\
\text { G-3 }\end{array}$ & $\begin{array}{l}\text { MGW } \\
\text { W-7 }\end{array}$ & $\begin{array}{l}\text { MTM } \\
\text { M-5 }\end{array}$ & $\begin{array}{l}\text { MTNU } \\
-5\end{array}$ & $\begin{array}{c}\text { NEKP- } \\
3\end{array}$ & $\begin{array}{c}\text { NEMR } \\
-3\end{array}$ \\
\hline $\begin{array}{c}\text { 1. Plant } \\
\text { habit }\end{array}$ & $\mathrm{D}$ & IM & $\mathrm{IM}$ & IM & E & IM & IM & IM & IM & E & $\mathrm{E}$ & IM & $\mathrm{E}$ \\
\hline $\begin{array}{l}\text { 2. Nature } \\
\text { of the } \\
\text { plant }\end{array}$ & Robust & $\begin{array}{l}\text { Mediu } \\
\mathrm{m}\end{array}$ & $\begin{array}{l}\text { Mediu } \\
\text { m }\end{array}$ & $\begin{array}{l}\text { Mediu } \\
\text { m }\end{array}$ & $\begin{array}{l}\text { Mediu } \\
\text { m }\end{array}$ & $\begin{array}{l}\text { Mediu } \\
\text { m }\end{array}$ & $\begin{array}{l}\text { Mediu } \\
\text { m }\end{array}$ & Small & Robust & Robust & $\begin{array}{l}\text { Mediu } \\
\text { m }\end{array}$ & $\begin{array}{c}\text { Mediu } \\
\text { m }\end{array}$ & Small \\
\hline $\begin{array}{l}\text { 3. Plant } \\
\text { height } \\
\text { (cm) }\end{array}$ & 17 & 10.3 & 12.5 & 13.5 & 8.8 & 13.5 & 6.5 & 15 & 13 & 9 & 10.7 & 25 & 7.5 \\
\hline $\begin{array}{l}\text { 4.Number } \\
\text { of leaflets }\end{array}$ & 5 & 3 & 3 & 3 & 5 & 3 & 9/11 & 3 & 7 & $5 / 7$ & 5 & 3 & 3 \\
\hline $\begin{array}{l}\text { 5. Stem } \\
\text { trichome* }\end{array}$ & Present & Present & Present & Present & Present & Present & Present & Present & Present & Present & Present & Present & Present \\
\hline $\begin{array}{c}6 . \\
\text { Phyllotax } \\
\mathrm{y}^{*} \\
\end{array}$ & $\begin{array}{c}\text { Whorle } \\
\text { d }\end{array}$ & $\begin{array}{c}\text { Whorle } \\
\text { d }\end{array}$ & $\begin{array}{c}\text { Whorle } \\
\text { d }\end{array}$ & $\begin{array}{c}\text { Whorle } \\
\text { d }\end{array}$ & $\begin{array}{c}\text { Whorle } \\
\text { d }\end{array}$ & $\begin{array}{c}\text { Whorle } \\
\text { d }\end{array}$ & $\begin{array}{c}\text { Whorle } \\
\text { d }\end{array}$ & $\begin{array}{l}\text { Whorle } \\
\text { d }\end{array}$ & $\begin{array}{l}\text { Whorle } \\
\text { d }\end{array}$ & $\begin{array}{c}\text { Whorle } \\
\text { d }\end{array}$ & $\begin{array}{l}\text { Whorle } \\
\text { d }\end{array}$ & $\begin{array}{c}\text { Whorle } \\
\text { d }\end{array}$ & $\begin{array}{c}\text { Whorle } \\
\text { d }\end{array}$ \\
\hline $\begin{array}{c}\text { 7. Leaf } \\
\text { venation* }\end{array}$ & PR & PR & PR & PR & PR & PR & PR & PR & PR & PR & PR & PR & PR \\
\hline $\begin{array}{l}\text { 8. Leaf } \\
\text { texture }\end{array}$ & Velvet & Velvet & $\begin{array}{c}\text { Smoot } \\
\text { h shiny }\end{array}$ & $\begin{array}{c}\text { Smoot } \\
\text { h shiny }\end{array}$ & Rough & $\begin{array}{c}\text { Smoot } \\
\text { h shiny }\end{array}$ & Rough & Rough & Rough & $\begin{array}{c}\text { Smoot } \\
\text { h shiny }\end{array}$ & Rough & Velvet & Velvet \\
\hline $\begin{array}{l}\text { 9. Leaf } \\
\text { type* }\end{array}$ & IP & IP & IP & IP & IP & IP & IP & IP & IP & IP & IP & IP & IP \\
\hline $\begin{array}{l}10 . \\
\text { Petiole } \\
\text { length } \\
(\mathrm{cm})\end{array}$ & 3.5 & 7.5 & 6 & 5.5 & 5 & 6 & 2 & 4.5 & 4 & 8 & 3.5 & 3.5 & 2.5 \\
\hline $\begin{array}{l}\text { 11. Leaf } \\
\text { length } \\
(\mathrm{cm})\end{array}$ & 12 & 18 & 15 & 14 & 7 & 18 & 17.5 & 9.5 & 12 & 15 & 9 & 10 & 7.5 \\
\hline $\begin{array}{l}\text { 12. Leaf } \\
\text { width } \\
(\mathrm{cm})\end{array}$ & 9 & 4.5 & 5 & 5 & 8 & 5 & 8 & 4 & 3 & 4.5 & 7 & 8 & 4.5 \\
\hline $\begin{array}{l}13 \text { Leaf } \\
\text { L/W ratio }\end{array}$ & 1.3 & 4 & 3 & 2.8 & 1.1 & 3.6 & 2.2 & 2.3 & 4 & 3.3 & 1.2 & 1.25 & 1.6 \\
\hline $\begin{array}{c}\text { 14. Termin } \\
\text { al leaflet } \\
\text { length } \\
(\mathrm{cm})\end{array}$ & 4.5 & 8 & 7 & 7.5 & 4.5 & 9 & 7 & 4 & 4.5 & 7 & 5.5 & 6 & 4 \\
\hline $\begin{array}{l}\text { 15. T.leaf } \\
\text { width } \\
\text { (cm) }\end{array}$ & 2 & 2.5 & 2.5 & 2.5 & 2.5 & 3.5 & 3.2 & 2.5 & 3 & 2.5 & 2 & 2.5 & 2 \\
\hline $\begin{array}{c}16 \mathrm{~T} . \\
\text { leaf . } \mathrm{L} \\
/ \mathrm{W} \text { ratio }\end{array}$ & 2.2 & 3.2 & 2.8 & 3 & 1.8 & 2.5 & 2.2 & 1.6 & 1.5 & 2.8 & 2.7 & 2.4 & 2 \\
\hline $\begin{array}{c}17 . \\
\text { Terminal } \\
\text { leaf apex }\end{array}$ & Obtuse & Acute & Acute & Acute & Obtuse & Obtuse & Obtuse & Obtuse & Obtuse & Acute & Acute & Acute & Obtuse \\
\hline $\begin{array}{l}\text { 18. T. } \\
\text { leaflet } \\
\text { base* }\end{array}$ & $\begin{array}{l}\text { Round } \\
\text { ed }\end{array}$ & $\begin{array}{l}\text { Round } \\
\text { ed }\end{array}$ & $\begin{array}{l}\text { Round } \\
\text { ed }\end{array}$ & $\begin{array}{c}\text { Round } \\
\text { ed }\end{array}$ & $\begin{array}{c}\text { Round } \\
\text { ed }\end{array}$ & $\begin{array}{l}\text { Round } \\
\text { ed }\end{array}$ & $\begin{array}{l}\text { Round } \\
\text { ed }\end{array}$ & $\begin{array}{c}\text { Round } \\
\text { ed }\end{array}$ & $\begin{array}{l}\text { Round } \\
\text { ed }\end{array}$ & $\begin{array}{c}\text { Round } \\
\text { ed }\end{array}$ & $\begin{array}{l}\text { Round } \\
\text { ed }\end{array}$ & $\begin{array}{c}\text { Round } \\
\text { ed }\end{array}$ & $\begin{array}{l}\text { Round } \\
\text { ed }\end{array}$ \\
\hline
\end{tabular}




\begin{tabular}{|c|c|c|c|c|c|c|c|c|c|c|c|c|c|}
\hline $\begin{array}{c}\text { 19. Termin } \\
\text { al leaflet } \\
\text { margin }\end{array}$ & $\begin{array}{l}\text { Undula } \\
\text { te }\end{array}$ & Entire & $\begin{array}{l}\text { Entire } \\
\text { end } \\
\text { leaf } \\
\text { lobed }\end{array}$ & Entire & $\begin{array}{c}\text { Dentat } \\
\text { e end } \\
\text { leaf } \\
\text { lobed } \\
\end{array}$ & $\begin{array}{c}\text { Dentat } \\
\text { e end } \\
\text { leaf } \\
\text { lobed } \\
\end{array}$ & $\begin{array}{c}\text { Dentat } \\
\text { e end } \\
\text { leaf } \\
\text { lobed } \\
\end{array}$ & $\begin{array}{c}\text { Dentat } \\
\text { e end } \\
\text { leaf } \\
\text { lobed } \\
\end{array}$ & $\begin{array}{c}\text { Dentat } \\
\text { e end } \\
\text { leaf } \\
\text { lobed } \\
\end{array}$ & Entire & $\begin{array}{c}\text { Dentat } \\
\text { e end } \\
\text { leaf } \\
\text { lobed } \\
\end{array}$ & $\begin{array}{c}\text { Dentat } \\
\text { e end } \\
\text { leaf } \\
\text { lobed } \\
\end{array}$ & Entire \\
\hline $\begin{array}{c}\text { 20.Numbe } \\
\mathrm{r} \text { of veins } \\
\text { in } \\
\text { Terminal } \\
\text { leaflet }\end{array}$ & 14 & 14 & 14 & 12 & 10 & 11 & 10 & 8 & 10 & 10 & 10 & 10 & 12 \\
\hline $\begin{array}{c}21 . \\
\text { Proximal } \\
\text { leaflet } \\
\text { length } \\
(\mathrm{cm})\end{array}$ & 3 & 5 & 3 & 3 & 3 & 3 & 3 & 3 & 3 & 3 & 3 & 3 & 3 \\
\hline $\begin{array}{c}\text { CHARAC } \\
\text { TER }\end{array}$ & $\begin{array}{l}\text { APRG- } \\
5 \\
\end{array}$ & $\begin{array}{c}\text { BDHM } \\
-3 \\
\end{array}$ & $\begin{array}{c}\text { GPPW } \\
-3 \\
\end{array}$ & $\begin{array}{c}\text { GPWP } \\
-3 \\
\end{array}$ & $\begin{array}{c}\text { KGKP } \\
-5 \\
\end{array}$ & $\begin{array}{c}\text { MGM } \\
\text { D-3 }\end{array}$ & $\begin{array}{c}\text { MGM } \\
\text { G-9 }\end{array}$ & $\begin{array}{c}\text { MGN } \\
\text { G-3 }\end{array}$ & $\begin{array}{c}\text { MGW } \\
\text { W-7 }\end{array}$ & $\begin{array}{c}\text { MTM } \\
\text { M-5 }\end{array}$ & $\begin{array}{c}\text { MTNU } \\
-5\end{array}$ & $\begin{array}{c}\text { NEKP- } \\
3 \\
\end{array}$ & $\begin{array}{c}\text { NEMR } \\
-3\end{array}$ \\
\hline $\begin{array}{c}22 . \\
\text { Proximal } \\
\text { leaflet } \\
\text { width } \\
(\mathrm{cm}) \\
\end{array}$ & 1.5 & 3 & 1.5 & 1 & 1.2 & 2 & 1.5 & 1.5 & 2 & 1.5 & 1.7 & 1.2 & 2.5 \\
\hline $\begin{array}{l}23 \text { P. leaf } \\
\text { L/W ratio }\end{array}$ & 2 & 1.6 & 2 & 3 & 2.5 & 1.5 & 2 & 2 & 1.5 & 2 & 1.7 & 2.5 & 1.2 \\
\hline $\begin{array}{c}24 \\
\text { Proximal } \\
\text { leaflet } \\
\text { apex }\end{array}$ & Obtuse & Acute & Acute & Acute & Obtuse & Acute & Acute & Obtuse & Obtuse & Acute & Acute & Obtuse & Acute \\
\hline $\begin{array}{c}25 . \\
\text { Proximal } \\
\text { leaflet } \\
\text { base } \\
\end{array}$ & $\begin{array}{l}\text { Obliqu } \\
\text { e }\end{array}$ & $\begin{array}{l}\text { Round } \\
\text { ed }\end{array}$ & $\begin{array}{l}\text { Round } \\
\text { ed }\end{array}$ & $\begin{array}{l}\text { Round } \\
\text { ed }\end{array}$ & $\begin{array}{l}\text { Obliqu } \\
\mathrm{e}\end{array}$ & $\begin{array}{l}\text { Round } \\
\text { ed }\end{array}$ & $\begin{array}{l}\text { Obliqu } \\
\text { e }\end{array}$ & $\begin{array}{l}\text { Round } \\
\text { ed }\end{array}$ & $\begin{array}{l}\text { Round } \\
\text { ed }\end{array}$ & $\begin{array}{l}\text { Obliqu } \\
\text { e }\end{array}$ & $\begin{array}{l}\text { Obliqu } \\
\text { e }\end{array}$ & $\begin{array}{l}\text { Obliqu } \\
\mathrm{e}\end{array}$ & $\begin{array}{c}\text { Obliqu } \\
\mathrm{e}\end{array}$ \\
\hline $\begin{array}{c}26 \\
\text { Proximal } \\
\text { leaflet } \\
\text { margin }\end{array}$ & $\begin{array}{l}\text { Crenat } \\
\text { e }\end{array}$ & Entire & $\begin{array}{l}\text { Undula } \\
\text { te }\end{array}$ & $\begin{array}{l}\text { Undula } \\
\text { te }\end{array}$ & $\begin{array}{l}\text { Dentat } \\
\text { e }\end{array}$ & $\begin{array}{l}\text { Dentat } \\
\text { e }\end{array}$ & $\begin{array}{l}\text { Dentat } \\
\text { e }\end{array}$ & $\begin{array}{l}\text { Dentat } \\
\text { e }\end{array}$ & $\begin{array}{l}\text { Dentat } \\
\text { e }\end{array}$ & $\begin{array}{l}\text { Undula } \\
\text { te }\end{array}$ & $\begin{array}{l}\text { Dentat } \\
\text { e }\end{array}$ & $\begin{array}{l}\text { Dentat } \\
\text { e }\end{array}$ & Entire \\
\hline $\begin{array}{c}27 . \\
\text { Proximal } \\
\text { leaflet } \\
\text { petiolule }\end{array}$ & NP & $\mathrm{P}$ & $\mathrm{P}$ & $\mathrm{P}$ & $\mathrm{P}$ & NP & NP & NP & NP & $\mathrm{P}$ & NP & $\mathrm{P}$ & $\mathrm{P}$ \\
\hline $\begin{array}{c}28 \text { No. } \\
\text { veins in } \\
\text { proximal } \\
\text { leaflet }\end{array}$ & 8 & 10 & 6 & 6 & 8 & 8 & 8 & 8 & 8 & 10 & 8 & 8 & 8 \\
\hline $\begin{array}{c}29 . \\
\text { Stipules* }\end{array}$ & Absent & Absent & Absent & Absent & Absent & Absent & Absent & Absent & Absent & Absent & Absent & Absent & Absent \\
\hline $\begin{array}{c}\text { 30. Leaf } \\
\text { trichomes } \\
*\end{array}$ & Present & Present & Present & Present & Present & Present & Present & Present & Present & Present & Present & Present & Present \\
\hline $\begin{array}{l}\text { 31. P-S } \\
\text { angle(a) }\end{array}$ & $45-60$ & $45-60$ & $45-60$ & $45-60$ & $45-60$ & $45-60$ & $<45$ & $45-60$ & $45-60$ & $45-60$ & $45-60$ & $45-60$ & $45-60$ \\
\hline $\begin{array}{l}\text { 32. P-S } \\
\text { angle (b) }\end{array}$ & $<45$ & $<45$ & $45-60$ & $45-60$ & $45-60$ & $<45$ & $<45$ & $<45$ & $45-60$ & $<45$ & $<45$ & $45-60$ & $<45$ \\
\hline $\begin{array}{c}\text { 33. S-T } \\
\text { angle }\end{array}$ & $60-90$ & $60-90$ & $60-90$ & $60-90$ & $60-90$ & $60-90$ & $60-90$ & 90 & 60-90 & $45-60$ & 60-90 & $60-90$ & $60-90$ \\
\hline \multicolumn{14}{|l|}{$\begin{array}{c}\text { Reproduct } \\
\text { ive } \\
\text { characters }\end{array}$} \\
\hline $\begin{array}{c}\text { 34. No. of } \\
\text { flowers in } \\
\text { infloresce } \\
\text { nt }\end{array}$ & 4 & 2 & 2 & 2 & 2 & 3 & 2 & 3 & 2 & 3 & 2 & 6 & 2 \\
\hline $\begin{array}{c}35 . \\
\text { Corolla } \\
\text { type* }\end{array}$ & S & S & S & S & S & S & $S$ & S & S & S & S & $S$ & S \\
\hline $\begin{array}{c}36 . \\
\text { Flower } \\
\text { colour* }\end{array}$ & White & White & White & White & White & White & White & White & White & White & White & White & White \\
\hline $\begin{array}{c}37 . \\
\text { Flower } \\
\text { symmetry } \\
*\end{array}$ & $\mathrm{AM}$ & AM & AM & $\mathrm{AM}$ & $\mathrm{AM}$ & $\mathrm{AM}$ & $\mathrm{AM}$ & AM & AM & $\mathrm{AM}$ & $\mathrm{AM}$ & $\mathrm{AM}$ & $\mathrm{AM}$ \\
\hline $\begin{array}{l}\text { 38.Coroll } \\
\text { a length } \\
(\mathrm{cm})\end{array}$ & 2.4 & 2.8 & 3 & 2.2 & 2.5 & 2 & 3.0 & 2 & 2.6 & 3 & 3 & 2.5 & 2.5 \\
\hline \multirow[t]{2}{*}{$\begin{array}{c}\text { 39.Pedicel } \\
*\end{array}$} & Present & Present & Present & Present & Present & Present & Present & Present & Present & Present & Present & Present & Present \\
\hline & APRG- & BDHM & GPPW & GPWP & KGKP & MGM & MGM & MGN & MGW & MTM & MTNU & NEKP- & NEMR \\
\hline
\end{tabular}




\begin{tabular}{|c|c|c|c|c|c|c|c|c|c|c|c|c|c|}
\hline \multirow{2}{*}{\begin{tabular}{|c|} 
Reproduct \\
ive \\
characters
\end{tabular}} & 5 & -3 & -3 & -3 & -5 & D-3 & G-9 & G-3 & W-7 & M-5 & -5 & 3 & -3 \\
\hline & & & & & & & & & & & & & \\
\hline $\begin{array}{l}\text { 40. No. of } \\
\text { sepals* }\end{array}$ & 5 & 5 & 5 & 5 & 5 & 5 & 5 & 5 & 5 & 5 & 5 & 5 & 5 \\
\hline $\begin{array}{c}\text { 41. Sepal } \\
\text { trichome } \\
\text { (u)* }\end{array}$ & Present & Present & Present & Present & Present & Present & Present & Present & Present & Present & Present & Present & Present \\
\hline $\begin{array}{c}\text { 42.Sepal } \\
\text { length(m } \\
\mathrm{m})\end{array}$ & 5 & 7 & 7 & 7 & 5 & 5 & 6 & 4 & 5 & 7 & 6 & 5 & 3 \\
\hline $\begin{array}{l}\text { 43. Sepal } \\
\text { width } \\
(\mathrm{mm})\end{array}$ & 1 & 1 & 1 & 1 & 1 & 1 & 2 & 1.5 & 1 & 1 & 1 & 1 & 1 \\
\hline $\begin{array}{l}44 \text {. Sepal } \\
\text { L/W ratio }\end{array}$ & 5 & 7 & 7 & 7 & 5 & 5 & 3 & 2.2 & 5 & 7 & 6 & 5 & 3 \\
\hline $\begin{array}{c}\text { 45. Sepal } \\
\text { trichomes } \\
(1)^{*}\end{array}$ & Present & Present & Present & Present & Present & Present & Present & Present & Present & Present & Present & Present & Present \\
\hline $\begin{array}{l}\text { 46. No. of } \\
\text { petal* }^{*}\end{array}$ & 5 & 5 & 5 & 5 & 5 & 5 & 5 & 5 & 5 & 5 & 5 & 5 & 5 \\
\hline $\begin{array}{c}\text { 47. Petal } \\
\text { length }(\mathrm{cm} \\
)\end{array}$ & 1.5 & 2 & 1.7 & 2 & 2.1 & 1.5 & 2 & 1.3 & 2.2 & 2 & 2.1 & 1.8 & 1.2 \\
\hline $\begin{array}{c}\text { 48. Petal } \\
\text { width }(\mathrm{cm})\end{array}$ & 0.4 & 0.7 & 0.7 & 1 & 0.6 & 0.5 & 0.5 & 0.4 & 1 & 0.9 & 0.7 & 1.0 & 0.4 \\
\hline $\begin{array}{c}49 \text { Petal } \\
\text { L/W ratio }\end{array}$ & 3.75 & 2.85 & 2.42 & 2 & 3.5 & 3 & 4 & 3.25 & 2.2 & 2.2 & 3 & 1.8 & 3 \\
\hline $\begin{array}{c}50 . \\
\text { Flower } \\
\text { length } \\
(\mathrm{cm}) \\
\end{array}$ & 3.2 & 4.5 & 3 & 2.9 & 2.6 & 2.8 & 3.5 & 3 & 2.8 & 3.2 & 4.3 & 3 & 2.5 \\
\hline $\begin{array}{c}\text { 51.Petal } \\
\text { apex }\end{array}$ & Acute & Acute & Acute & Acute & $\begin{array}{c}\text { Round } \\
\text { ed }\end{array}$ & Acute & Acute & $\begin{array}{c}\text { Trunca } \\
\text { te }\end{array}$ & $\begin{array}{c}\text { Round } \\
\text { ed }\end{array}$ & Acute & Acute & Acute & Acute \\
\hline $\begin{array}{c}\text { 52.Petal } \\
\text { margin } \\
\text { trichomes } \\
(\mathrm{u}) .^{*}\end{array}$ & Present & Present & Present & Present & Present & Present & Present & Present & Present & Present & Present & Present & Present \\
\hline $\begin{array}{l}\text { 53. Petal } \\
\text { trichomes } \\
(1)^{*}\end{array}$ & Dense & Dense & Dense & Dense & Dense & Dense & Dense & Dense & Dense & Dense & Dense & Dense & Dense \\
\hline $\begin{array}{l}\text { 54. Petal } \\
\text { tri (u) }\end{array}$ & Absent & Absent & Absent & Absent & Absent & Absent & Absent & Absent & Absent & Absent & Absent & Absent & Absent \\
\hline $\begin{array}{c}\text { 55.Petal } \\
\text { venation* }\end{array}$ & PR & PR & PR & PR & PR & PR & PR & PR & PR & PR & PR & PR & PR \\
\hline & $\begin{array}{c}\text { APRG- } \\
5 \\
\end{array}$ & $\begin{array}{c}\text { BDHM } \\
-3 \\
\end{array}$ & $\begin{array}{c}\text { GPPW } \\
-3 \\
\end{array}$ & $\begin{array}{c}\text { GPWP } \\
-3 \\
\end{array}$ & $\begin{array}{c}\text { KGKP } \\
-5 \\
\end{array}$ & $\begin{array}{c}\text { MGM } \\
\text { D-3 } \\
\end{array}$ & $\begin{array}{c}\text { MGM } \\
\text { G-9 }\end{array}$ & $\begin{array}{c}\text { MGN } \\
\text { G-3 }\end{array}$ & $\begin{array}{c}\text { MGW } \\
\text { W-7 }\end{array}$ & $\begin{array}{c}\text { MTM } \\
\text { M-5 } \\
\end{array}$ & $\begin{array}{c}\text { MTNU } \\
-5 \\
\end{array}$ & $\begin{array}{c}\text { NEKP- } \\
3 \\
\end{array}$ & $\begin{array}{c}\text { NEMR } \\
-3 \\
\end{array}$ \\
\hline $\begin{array}{c}56 \\
\text { Androceiu } \\
\text { m type* }^{*}\end{array}$ & MD & MD & MD & MD & MD & MD & MD & MD & MD & MD & MD & MD & MD \\
\hline $\begin{array}{c}\text { 57.Stamin } \\
\text { a tube* }\end{array}$ & Present & Present & Present & Present & Present & Present & Present & Present & Present & Present & Present & Present & Present \\
\hline $\begin{array}{c}\text { 58.Stamin } \\
\text { al tube L } \\
(\mathrm{cm})\end{array}$ & 2.3 & 2.5 & $\begin{array}{c}2.3 \\
\text { change } \\
\text { this }\end{array}$ & 2.5 & 2.4 & 2 & 2.7 & 2 & 2.5 & 2.9 & 2.5 & 2.0 & 2.0 \\
\hline $\begin{array}{c}59 . \\
\text { Anther fil. } \\
\text { Attachme } \\
\text { nt* }^{*}\end{array}$ & DF & DF & DF & DF & DF & DF & DF & DF & DF & $\mathrm{DF}$ & DF & DF & DF \\
\hline $\begin{array}{l}\text { 60.Anther } \\
\text { collar } \\
\text { attachmen } \\
\mathrm{t} * \\
\end{array}$ & DC & DC & DC & DC & DC & DC & DC & DC & DC & DC & DC & DC & DC \\
\hline $\begin{array}{c}\text { 61.Style } \\
\text { length }\end{array}$ & 2.4 & 2.6 & 2 & 2.1 & 2.5 & 2.2 & 2 & 2 & 2.9 & 3 & 2.9 & 3 & 2 \\
\hline $\begin{array}{c}\text { 62Anther } \\
\text { apex }\end{array}$ & $\begin{array}{l}\text { Mucro } \\
\text { nate }\end{array}$ & $\begin{array}{c}\text { Mucro } \\
\text { nate }\end{array}$ & $\begin{array}{l}\text { Mucro } \\
\text { nate }\end{array}$ & $\begin{array}{c}\text { Mucro } \\
\text { nate }\end{array}$ & $\begin{array}{l}\text { Mucro } \\
\text { nate }\end{array}$ & $\begin{array}{c}\text { Mucro } \\
\text { nate }\end{array}$ & $\begin{array}{c}\text { Mucro } \\
\text { nate }\end{array}$ & $\begin{array}{c}\text { Mucro } \\
\text { nate }\end{array}$ & $\begin{array}{c}\text { Mucro } \\
\text { nate }\end{array}$ & $\begin{array}{c}\text { Mucro } \\
\text { nate }\end{array}$ & $\begin{array}{c}\text { Mucro } \\
\text { nate }\end{array}$ & $\begin{array}{c}\text { Mucro } \\
\text { nate }\end{array}$ & $\begin{array}{c}\text { Mucro } \\
\text { nate }\end{array}$ \\
\hline $\begin{array}{l}\text { 63.Stigma } \\
\text { shape* }^{*}\end{array}$ & $\begin{array}{c}\text { Globos } \\
\mathrm{e}\end{array}$ & $\begin{array}{c}\text { Globos } \\
\mathrm{e}\end{array}$ & $\begin{array}{c}\text { Globos } \\
\text { e }\end{array}$ & $\begin{array}{c}\text { Globos } \\
\mathrm{e}\end{array}$ & $\begin{array}{c}\text { Globos } \\
\text { e }\end{array}$ & $\begin{array}{c}\text { Globos } \\
\mathrm{e}\end{array}$ & $\begin{array}{c}\text { Globos } \\
\text { e }\end{array}$ & $\begin{array}{c}\text { Globos } \\
\mathrm{e}\end{array}$ & $\begin{array}{c}\text { Globos } \\
\mathrm{e}\end{array}$ & $\begin{array}{c}\text { Globos } \\
\mathrm{e}\end{array}$ & $\begin{array}{c}\text { Globos } \\
\mathrm{e}\end{array}$ & $\begin{array}{c}\text { Globos } \\
\mathrm{e}\end{array}$ & $\begin{array}{c}\text { Globos } \\
\mathrm{e}\end{array}$ \\
\hline $\begin{array}{c}\text { 64.Style } \\
\text { base }\end{array}$ & $\begin{array}{c}\text { Globos } \\
\mathrm{e}\end{array}$ & Flat & Flat & Flat & Flat & Flat & Flat & Flat & Flat & Flat & Flat & Flat & Flat \\
\hline $\begin{array}{c}65 . \\
\text { Stigma } \\
\text { hairs* }\end{array}$ & Present & Present & Present & Present & Present & Present & Present & Present & Present & Present & Present & Present & Present \\
\hline
\end{tabular}




\begin{tabular}{|c|c|c|c|c|c|c|c|c|c|c|c|c|c|}
\hline $\begin{array}{c}66 . \\
\text { Ovary* }\end{array}$ & $\begin{array}{l}\text { Superi } \\
\text { or }\end{array}$ & $\begin{array}{l}\text { Superi } \\
\text { or }\end{array}$ & $\begin{array}{l}\text { Superi } \\
\text { or }\end{array}$ & $\begin{array}{l}\text { Superi } \\
\text { or }\end{array}$ & $\begin{array}{l}\text { Superi } \\
\text { or }\end{array}$ & $\begin{array}{l}\text { Superi } \\
\text { or }\end{array}$ & $\begin{array}{l}\text { Superi } \\
\text { or }\end{array}$ & $\begin{array}{l}\text { Superi } \\
\text { or }\end{array}$ & $\begin{array}{l}\text { Superi } \\
\text { or }\end{array}$ & $\begin{array}{l}\text { Superi } \\
\text { or }\end{array}$ & $\begin{array}{l}\text { Superi } \\
\text { or }\end{array}$ & $\begin{array}{l}\text { Superi } \\
\text { or }\end{array}$ & $\begin{array}{l}\text { Superi } \\
\text { or }\end{array}$ \\
\hline $\begin{array}{l}\text { 67. No. of } \\
\text { Carpels* }\end{array}$ & 5 & 5 & 5 & 5 & 5 & 5 & 5 & 5 & 5 & 5 & 5 & 5 & 5 \\
\hline \multirow[t]{2}{*}{$\begin{array}{c}68 . \\
\text { Placentati } \\
\text { on* }\end{array}$} & $\begin{array}{c}\text { Axillar } \\
\text { y }\end{array}$ & $\begin{array}{c}\text { Axillar } \\
\text { y }\end{array}$ & $\begin{array}{c}\text { Axillar } \\
\text { y }\end{array}$ & $\begin{array}{c}\text { Axillar } \\
\mathrm{y}\end{array}$ & $\begin{array}{c}\text { Axillar } \\
\text { y }\end{array}$ & $\begin{array}{c}\text { Axillar } \\
\text { y }\end{array}$ & $\begin{array}{c}\text { Axillar } \\
\text { y }\end{array}$ & $\begin{array}{c}\text { Axillar } \\
y\end{array}$ & $\begin{array}{c}\text { Axillar } \\
\mathrm{y}\end{array}$ & $\begin{array}{c}\text { Axillar } \\
\text { y }\end{array}$ & $\begin{array}{c}\text { Axillar } \\
\mathrm{y}\end{array}$ & $\begin{array}{c}\text { Axillar } \\
\text { y }\end{array}$ & $\begin{array}{c}\text { Axillar } \\
\mathrm{y}\end{array}$ \\
\hline & $\begin{array}{l}\text { APRG- } \\
5\end{array}$ & $\begin{array}{c}\text { BDHM } \\
-3\end{array}$ & $\begin{array}{c}\text { GPPW } \\
-3\end{array}$ & $\begin{array}{c}\text { GPWP } \\
-3\end{array}$ & $\begin{array}{c}\text { KGKP } \\
-5\end{array}$ & $\begin{array}{c}\text { MGM } \\
\text { D-3 }\end{array}$ & $\begin{array}{c}\text { MGM } \\
\text { G-9 }\end{array}$ & $\begin{array}{c}\text { MGN } \\
\text { G-3 }\end{array}$ & $\begin{array}{c}\text { MGW } \\
\text { W-7 }\end{array}$ & $\begin{array}{c}\text { MTM } \\
\text { M-5 }\end{array}$ & $\begin{array}{c}\text { MTNU } \\
-5\end{array}$ & $\begin{array}{c}\text { NEKP- } \\
3\end{array}$ & $\begin{array}{c}\text { NEMR } \\
-3\end{array}$ \\
\hline $\begin{array}{l}\text { 69. Fruit } \\
\text { type* }\end{array}$ & $\begin{array}{c}\text { Capsul } \\
\text { e }\end{array}$ & $\begin{array}{c}\text { Capsul } \\
\mathrm{e}\end{array}$ & $\begin{array}{c}\text { Capsul } \\
\mathrm{e}\end{array}$ & $\begin{array}{c}\text { Capsul } \\
\mathrm{e}\end{array}$ & $\begin{array}{c}\text { Capsul } \\
\mathrm{e}\end{array}$ & $\begin{array}{c}\text { Capsul } \\
\text { e }\end{array}$ & $\begin{array}{c}\text { Capsul } \\
\mathrm{e}\end{array}$ & $\begin{array}{c}\text { Capsul } \\
\text { e }\end{array}$ & $\begin{array}{c}\text { Capsul } \\
\mathrm{e}\end{array}$ & $\begin{array}{c}\text { Capsul } \\
\mathrm{e}\end{array}$ & $\begin{array}{c}\text { Capsul } \\
\text { e }\end{array}$ & $\begin{array}{c}\text { Capsul } \\
\mathrm{e}\end{array}$ & $\begin{array}{c}\text { Capsul } \\
\text { e }\end{array}$ \\
\hline $\begin{array}{l}\text { 70. Seed } \\
\text { No. }\end{array}$ & 5 & 8 & 7 & 8 & 5 & 7 & 9 & 8 & 5 & 8 & 6 & 10 & 8 \\
\hline $\begin{array}{l}\text { 71. Seed } \\
\text { colour* }\end{array}$ & Brown & Brown & Brown & Brown & Brown & Brown & Brown & Brown & Brown & Brown & Brown & Brown & Brown \\
\hline $\begin{array}{l}72 \text { Seed } \\
\text { length } \\
(\mathrm{mm})\end{array}$ & 4 & 4 & 4 & 4 & 4 & 4.5 & 4 & 4 & 4 & 4 & 4 & 4 & 4 \\
\hline $\begin{array}{l}\text { 73.Seed } \\
\text { width } \\
(\mathrm{mm}) \\
\end{array}$ & 2.5 & 2.5 & 2 & 2.5 & 2 & 2 & 2 & 2 & 2 & 2.5 & 2.5 & 2.5 & 2 \\
\hline $\begin{array}{c}74 \text { Seed } \\
\text { L/W }\end{array}$ & 1.6 & 1.6 & 2 & 1.6 & 2 & 2.2 & 2 & 2 & 2 & 1.6 & 1.6 & 1.6 & 2 \\
\hline $\begin{array}{c}\text { 75. Areole } \\
\text { shape }\end{array}$ & PG & PG & PG & PG & PG & PG & PG & PG & PG & PG & PG & PG & PG \\
\hline $\begin{array}{l}\text { 76. Vein } \\
\text { islet No }\end{array}$ & 3.70 & 1.85 & 2.78 & 2.78 & 1.39 & 1.39 & 0.93 & 1.39 & 1.39 & 2.31 & 0.93 & 1.85 & 1.85 \\
\hline $\begin{array}{l}\text { 77. Vein } \\
\text { let termin. } \\
\text { No. }\end{array}$ & 7.41 & 3.70 & 6.45 & 6.48 & 3.24 & 2.78 & 3.24 & 3.24 & 3.24 & 5.09 & 3.24 & 3.70 & 3.70 \\
\hline
\end{tabular}

PG-Polygonal; P-Prominent; NP-Not prominent; IM-Intermediate; D-Droopy; E-Erect *Denotes the monomorphic characters; PR- Pinnately reticulate; MD- Monodelphous; DC-Double cordate; AM- Actinomorphic; S- Salverform; IP-Imparipinnate; P-S angle- Primary-secondary vein angle; S-T anglesecondary vein- tertiary vein angle; DF-Dorsifixed;

\section{Annexure 2}

Table 5. Numerical data table for cluster analysis of different populations of $M$. pinnata

\begin{tabular}{|c|c|c|c|c|c|c|c|c|c|c|c|c|c|}
\hline \multirow[b]{2}{*}{ Character } & \multicolumn{13}{|c|}{ Population } \\
\hline & $\begin{array}{l}\text { MGM } \\
\text { D-3 }\end{array}$ & $\begin{array}{l}\text { MGM } \\
\text { G9/11 }\end{array}$ & $\begin{array}{l}\text { GPW } \\
\text { P-3 }\end{array}$ & $\begin{array}{c}\text { NGN } \\
\text { G-3 }\end{array}$ & $\begin{array}{l}\text { NEM } \\
\text { R-3 }\end{array}$ & $\begin{array}{l}\mathrm{BDH} \\
\mathrm{M}-3\end{array}$ & $\begin{array}{l}\text { APR } \\
\text { G-5 }\end{array}$ & $\begin{array}{l}\text { MTM } \\
\text { M-5/7 }\end{array}$ & $\begin{array}{l}\text { NEK } \\
\text { P-3 }\end{array}$ & $\begin{array}{l}\text { MTN } \\
\text { U-5 }\end{array}$ & $\begin{array}{l}\text { GPP } \\
W-3\end{array}$ & $\begin{array}{l}\text { MGW } \\
\text { W-7 }\end{array}$ & $\begin{array}{c}\text { KGK } \\
\text { P-5 }\end{array}$ \\
\hline $\begin{array}{l}\text { a. Vegetative } \\
\text { characters }\end{array}$ & & & & & & & & & & & & & \\
\hline Plant habit & 3 & 3 & 1 & 3 & 2 & 3 & 1 & 2 & 3 & 2 & 3 & 3 & 2 \\
\hline $\begin{array}{c}\text { Plant size } \\
\text { (Nature) }\end{array}$ & 2 & 2 & 2 & 3 & 3 & 2 & 1 & 1 & 2 & 2 & 2 & 1 & 2 \\
\hline $\begin{array}{c}\text { Plant } \\
\text { height }(\mathrm{cm})\end{array}$ & 2 & 1 & 2 & 3 & 1 & 2 & 3 & 1 & 3 & 2 & 2 & 2 & 1 \\
\hline Leaflet no. & 1 & 4 & 1 & 1 & 1 & 1 & 2 & 3 & 1 & 2 & 1 & 3 & 2 \\
\hline Stem trichome & 1 & 1 & 1 & 1 & 1 & 1 & 1 & 1 & 1 & 1 & 1 & 1 & 1 \\
\hline Phyllotaxy & 1 & 1 & 1 & 1 & 1 & 1 & 1 & 1 & 1 & 1 & 1 & 1 & 1 \\
\hline Leaf venation & 1 & 1 & 1 & 1 & 1 & 1 & 1 & 1 & 1 & 1 & 1 & 1 & 1 \\
\hline Leaf texture & 2 & 3 & 4 & 3 & 1 & 1 & 1 & 2 & 1 & 3 & 2 & 3 & 3 \\
\hline Leaf form & 1 & 1 & 1 & 1 & 1 & 1 & 1 & 1 & 1 & 1 & 1 & 1 & 1 \\
\hline $\begin{array}{l}\text { Petiole length } \\
\text { (cm) }\end{array}$ & 2 & 1 & 2 & 2 & 1 & 3 & 1 & 3 & 1 & 1 & 2 & 1 & 2 \\
\hline $\begin{array}{l}\text { Leaf length } \\
\text { (cm) }\end{array}$ & 3 & 3 & 2 & 1 & 1 & 3 & 2 & 2 & 1 & 1 & 2 & 2 & 1 \\
\hline $\begin{array}{l}\text { Leaf width } \\
\text { (cm) }\end{array}$ & 1 & 3 & 1 & 1 & 1 & 1 & 3 & 1 & 3 & 2 & 1 & 1 & 2 \\
\hline $\begin{array}{c}\text { E.leaflet } \\
\text { length }(\mathrm{cm})\end{array}$ & 3 & 2 & 2 & 1 & 1 & 3 & 1 & 2 & 2 & 1 & 2 & 1 & 1 \\
\hline $\begin{array}{l}\text { E.leaflet width } \\
\text { (cm) }\end{array}$ & 2 & 2 & 1 & 1 & 1 & 1 & 1 & 1 & 1 & 1 & 1 & 2 & 1 \\
\hline $\begin{array}{c}\text { End leaflet } \\
\text { apex }\end{array}$ & 2 & 2 & 1 & 2 & 2 & 1 & 2 & 1 & 1 & 1 & 1 & 2 & 2 \\
\hline End leaflet base & 1 & 1 & 1 & 1 & 1 & 1 & 1 & 1 & 1 & 1 & 1 & 1 & 1 \\
\hline $\begin{array}{l}\text { End leaflet } \\
\text { margin }\end{array}$ & 2 & 2 & 1 & 2 & 1 & 1 & 3 & 3 & 1 & 2 & 3 & 2 & 2 \\
\hline $\begin{array}{c}\text { E leaflet vein } \\
\text { no. }\end{array}$ & 2 & 2 & 2 & 1 & 2 & 3 & 3 & 2 & 2 & 2 & 2 & 2 & 2 \\
\hline $\begin{array}{l}\text { Side leaflet } \\
\text { length }\end{array}$ & 1 & 1 & 1 & 1 & 1 & 1 & 2 & 1 & 1 & 1 & 2 & 1 & 1 \\
\hline $\begin{array}{l}\text { Side leaflet } \\
\text { width }\end{array}$ & 3 & 3 & 3 & 3 & 2 & 3 & 2 & 2 & 3 & 2 & 3 & 2 & 1 \\
\hline
\end{tabular}




\begin{tabular}{|c|c|c|c|c|c|c|c|c|c|c|c|c|c|}
\hline $\begin{array}{c}\text { Side leaflet } \\
\text { apex }\end{array}$ & 1 & 1 & 1 & 2 & 1 & 1 & 2 & 1 & 1 & 1 & 1 & 2 & 2 \\
\hline $\begin{array}{c}\text { Side leaflet } \\
\text { base }\end{array}$ & 1 & 2 & 1 & 1 & 2 & 1 & 2 & 2 & 1 & 2 & 1 & 1 & 2 \\
\hline $\begin{array}{l}\text { Side leaflet } \\
\text { margin }\end{array}$ & 2 & 2 & 3 & 2 & 1 & 1 & 4 & 3 & 1 & 2 & 3 & 2 & 2 \\
\hline $\begin{array}{l}\text { Side leaflet } \\
\text { petiolule }\end{array}$ & 2 & 2 & 1 & 2 & 1 & 1 & 2 & 1 & 1 & 2 & 1 & 2 & 1 \\
\hline $\begin{array}{l}\text { Side leaflet } \\
\text { vein no. }\end{array}$ & 2 & 2 & 1 & 2 & 2 & 3 & 2 & 3 & 2 & 2 & 1 & 2 & 2 \\
\hline Stipules & 2 & 2 & 2 & 2 & 2 & 2 & 2 & 2 & 2 & 2 & 2 & 2 & 2 \\
\hline Leaf Trichome & 1 & 1 & 1 & 1 & 1 & 1 & 1 & 1 & 1 & 1 & 1 & 1 & 1 \\
\hline P-S angle (a) & 2 & 1 & 2 & 2 & 2 & 2 & 2 & 2 & 2 & 2 & 2 & 2 & 2 \\
\hline P-S angle (b) & 1 & 1 & 2 & 1 & 1 & 1 & 1 & 1 & 2 & 1 & 2 & 2 & 2 \\
\hline S-T angle & 2 & 2 & 2 & 2 & 2 & 2 & 2 & 2 & 2 & 2 & 2 & 2 & 2 \\
\hline Epidermal cell & 3 & 1 & 3 & 2 & 3 & 1 & 1 & 2 & 3 & 3 & 1 & 1 & 1 \\
\hline Stomata index & 1 & 1 & 2 & 1 & 1 & 1 & 1 & 3 & 1 & 1 & 2 & 2 & 1 \\
\hline Sub. Cells & 1 & 2 & 2 & 2 & 2 & 2 & 2 & 2 & 2 & 2 & 2 & 2 & 2 \\
\hline \multicolumn{14}{|l|}{$\begin{array}{l}\text { b. Reproductive } \\
\text { characters }\end{array}$} \\
\hline $\begin{array}{l}\text { No. of flowers } \\
\text { in inflo. }\end{array}$ & 1 & 1 & 1 & 1 & 1 & 1 & 2 & 1 & 2 & 1 & 1 & 1 & 1 \\
\hline Corolla type & 1 & 1 & 1 & 1 & 1 & 1 & 1 & 1 & 1 & 1 & 1 & 1 & 1 \\
\hline $\begin{array}{l}\text { Flower colour } \\
\end{array}$ & 1 & 1 & 1 & 1 & 1 & 1 & 1 & 1 & 1 & 1 & 1 & 1 & 1 \\
\hline $\begin{array}{c}\text { Flower } \\
\text { symmetry }\end{array}$ & 1 & 1 & 1 & 1 & 1 & 1 & 1 & 1 & 1 & 1 & 1 & 1 & 1 \\
\hline $\begin{array}{l}\text { Corolla length } \\
\text { (cm) }\end{array}$ & 1 & 2 & 1 & 1 & 1 & 2 & 1 & 2 & 2 & 2 & 2 & 2 & 2 \\
\hline Pedicel & 1 & 1 & 1 & 1 & 1 & 1 & 1 & 1 & 1 & 1 & 1 & 1 & 1 \\
\hline No. of sepals & 1 & 1 & 1 & 1 & 1 & 1 & 1 & 1 & 1 & 1 & 1 & 1 & 1 \\
\hline $\begin{array}{l}\text { Sepal trichome } \\
\text { (u) }\end{array}$ & 1 & 1 & 1 & 1 & 1 & 1 & 1 & 1 & 1 & 1 & 1 & 1 & 1 \\
\hline $\begin{array}{l}\text { Sepal trichome } \\
\text { (l) }\end{array}$ & 1 & 1 & 1 & 1 & 1 & 1 & 1 & 1 & 1 & 1 & 1 & 1 & 1 \\
\hline $\begin{array}{l}\text { Sepal length } \\
\text { (mm) }\end{array}$ & 1 & 1 & 2 & 1 & 1 & 2 & 1 & 2 & 1 & 1 & 2 & 1 & 1 \\
\hline $\begin{array}{l}\text { Sepal width } \\
\text { (mm) }\end{array}$ & 1 & 2 & 1 & 1 & 1 & 1 & 1 & 1 & 1 & 1 & 2 & 1 & 1 \\
\hline No. of petal & 1 & 1 & 1 & 1 & 1 & 1 & 1 & 1 & 1 & 1 & 1 & 1 & 1 \\
\hline $\begin{array}{l}\text { Petal length } \\
\text { (cm) }\end{array}$ & 1 & 2 & 2 & 1 & 1 & 2 & 1 & 2 & 2 & 3 & 2 & 3 & 3 \\
\hline $\begin{array}{l}\text { Petal width } \\
\text { (cm) }\end{array}$ & 1 & 1 & 3 & 1 & 1 & 2 & 1 & 3 & 3 & 2 & 2 & 3 & 2 \\
\hline Petal apex & 1 & 1 & 1 & 2 & 1 & 1 & 1 & 1 & 1 & 1 & 1 & 2 & 2 \\
\hline Petal margin tri & 1 & 1 & 1 & 1 & 1 & 1 & 1 & 1 & 1 & 1 & 1 & 1 & 1 \\
\hline Petal tri lower & 1 & 1 & 1 & 1 & 1 & 1 & 1 & 1 & 1 & 1 & 1 & 1 & 1 \\
\hline Petal tri upper & 1 & 1 & 1 & 1 & 1 & 1 & 1 & 1 & 1 & 1 & 1 & 1 & 1 \\
\hline Petal venation & 1 & 1 & 1 & 1 & 1 & 1 & 1 & 1 & 1 & 1 & 1 & 1 & 1 \\
\hline $\begin{array}{l}\text { Androceium } \\
\text { type }\end{array}$ & 1 & 1 & 1 & 1 & 1 & 1 & 1 & 1 & 1 & 1 & 1 & 1 & 1 \\
\hline $\begin{array}{l}\text { Stamina tube L } \\
(\mathrm{cm})\end{array}$ & 1 & 2 & 2 & 1 & 1 & 2 & 1 & 2 & 1 & 2 & 1 & 1 & 1 \\
\hline $\begin{array}{l}\text { Anther fil. } \\
\text { Attach. }\end{array}$ & 1 & 1 & 1 & 1 & 1 & 1 & 1 & 1 & 1 & 1 & 1 & 1 & 1 \\
\hline $\begin{array}{l}\text { Anther collar } \\
\text { attach. }\end{array}$ & 1 & 1 & 1 & 1 & 1 & 1 & 1 & 1 & 1 & 1 & 1 & 1 & 1 \\
\hline Anther apex & 1 & 1 & 1 & 1 & 1 & 1 & 1 & 1 & 1 & 1 & 1 & 1 & 1 \\
\hline $\begin{array}{c}\text { Stigma } \\
\text { appendages }\end{array}$ & 1 & 1 & 1 & 1 & 1 & 1 & 1 & 1 & 1 & 1 & 1 & 1 & 1 \\
\hline Stigma shape & 1 & 1 & 1 & 1 & 1 & 1 & 1 & 1 & 1 & 1 & 1 & 1 & \\
\hline $\begin{array}{l}\text { Style length } \\
\text { (cm) }\end{array}$ & 2 & 3 & 2 & 1 & 1 & 3 & 2 & 3 & 1 & 3 & 3 & 3 & 2 \\
\hline Style base & 1 & 1 & 1 & 1 & 1 & 1 & 1 & 1 & 1 & 1 & 1 & 1 & 1 \\
\hline Stigma hairs & 2 & 2 & 2 & 2 & 2 & 2 & 2 & 2 & 2 & 2 & 2 & 2 & 2 \\
\hline Ovary & 1 & 1 & 1 & 1 & 1 & 1 & 1 & 1 & 1 & 1 & 1 & 1 & 1 \\
\hline Placentation & 1 & 1 & 1 & 1 & 1 & 1 & 1 & 1 & 1 & 1 & 1 & 1 & 1 \\
\hline Carpel No & 1 & 1 & 1 & 1 & 1 & 1 & 1 & 1 & 1 & 1 & 1 & 1 & 1 \\
\hline Fruit type & 1 & 1 & 1 & 1 & 1 & 1 & 1 & 1 & 1 & 1 & 1 & 1 & 1 \\
\hline Seed No. & 1 & 2 & 2 & 2 & 2 & 2 & 1 & 2 & 3 & 1 & 2 & 1 & 1 \\
\hline Seed colour & 1 & 1 & 1 & 1 & 1 & 1 & 1 & 1 & 1 & 1 & 1 & 1 & 1 \\
\hline $\begin{array}{l}\text { Seed length } \\
(\mathrm{mm})\end{array}$ & 2 & 1 & 1 & 1 & 1 & 1 & 1 & 1 & 1 & 1 & 1 & 1 & 1 \\
\hline $\begin{array}{l}\text { Seed width } \\
\text { (mm) }\end{array}$ & 1 & 1 & 2 & 1 & 1 & 2 & 2 & 2 & 2 & 2 & 1 & 1 & 1 \\
\hline
\end{tabular}

